\title{
Photosynthetic Pigments Profiled in Capsicum Lutescens Mutants
}

\author{
Calen McKenzie, Ivette Guzman, Ciro Velasco-Cruz, and Paul W. Bosland \\ Department of Plant and Environmental Sciences, New Mexico State University, 1780 E. \\ University Avenue, Las Cruces, NM 88003
}

\begin{abstract}
ADDiTIONAL INDEX wORDS. carotenoid, chlorophyll, chloroplast, chromoplast, principal component analysis
Abstract. Lutescens, or lutescent, plant mutants produce leaves that are abnormally light yellow-green compared with normal plants, and are observed in multiple species of Capsicum as well as other genera such as Zea, Oryza, and Oenothera. Previous investigations into the lutescent phenotype in Capsicum have focused on genetic and transcriptomic analyses, and comparatively little is known about the phytochemical constituents of the lutescent leaf phenotype. Previous research in similar lutescent mutants in Capsicum and Oryza species has attributed their pale yellowgreen leaf color and poor vigor to deficient chloroplast development. A total of 25 accessions of Capsicum lutescens mutants were phenotyped and analyzed based on a multivariate approach, using 'Jupiter' bell pepper (Capsicum annuиm) with normal green leaves as a contextual benchmark. Photosynthetic pigments from mutant leaves were extracted and analyzed using high-performance liquid chromatography (HPLC); reflectance of the leaf material was measured with a chromameter using the $L^{*} a^{*} b^{*}$ color space. The chlorophyll a $(\mathrm{Chl} a) / b(\mathrm{Chl} b)$ ratio was greater in leaves of lutescens mutants than in 'Jupiter'. Multivariate statistical analyses revealed all lutescent mutant accessions could be distinguished from the 'Jupiter' contextual benchmark by variables indicating poor chloroplast development and increased photooxidative stress in lutescent mutant accessions. The lutescent leaf phenotype was not found to be caused by elevated xanthophyll or decreased chlorophyll concentrations. Furthermore, multivariate analysis revealed the lutescent mutant phenotype to be variable, with a wide range of phenotypes clustered into four major groups.
\end{abstract}

Chlorophylls and carotenoids are responsible for harnessing the photon energy ultimately used in the splitting of a water molecule in photosynthesis (Buchanan, 2015). Two primary chlorophylls responsible for photosynthesis in plants - $\mathrm{Chl}$ a and $\mathrm{Chl}$ $\mathrm{b}$ - differ slightly in their absorption spectra. Carotenoids are important phytochemical pigments responsible for the color of most yellow, orange, and red fruits and vegetables. They are classified into two groups: carotenes and xanthophylls. Carotenes such as $\alpha$-carotene and $\beta$-carotene are pure hydrocarbons and are highly lipophilic (Buchanan, 2015). Xanthophylls, on the other hand, have one or more hydroxyl groups attached to one or both of the flanking rings, making them much less lipophilic than carotenes (Buchanan, 2015). In plants, carotenoids aid directly in photosynthesis, increasing the range of light absorption, acting as powerful antioxidants to quench singlet oxygen, and also providing valuable precursors for abscisic acid, which is a plant hormone vital to fruit maturity (Buchanan, 2015). Carotenoids have also been observed to aid in heat dissipation during high rates of photosynthesis (Ma et al., 2016). Capsicum species are widely used as excellent models for the

Received for publication 24 Nov. 2020. Accepted for publication 24 Feb. 2021

Published online 8 June 2021.

We thank Krystal Vargas, Danise Garcia, Rakhila Mamenova, and all the interns in the Crop Quality Lab at New Mexico State University (NMSU) for their technical assistance. We also thank Danise Coon, Sr. Research Specialist with the NMSU Chile Pepper Breeding Program and Chile Pepper Institute, for her integral assistance in the greenhouse, and Rich Richins for his technical guidance with the chromatography.

Graduate student salaries and research were supported by state and federal Hatch funds appropriated to the New Mexico Agricultural Experiment Station.

I.G. is the corresponding author. E-mail: ivguzman@nmsu.edu.

This is an open access article distributed under the CC BY-NC-ND license (https://creativecommons.org/licenses/by-nc-nd/4.0/). study of carotenoid biosynthesis because of their high accumulation of numerous carotenoids in the fruit (Gomez-Garcia and Ochoa-Alejo, 2013).

Lutescens, or lutescent, plant mutants produce leaves that are abnormally light yellow-green compared with standard plants and have been observed in multiple species of Capsicum and other genera such as Zea, Oryza, and Oenothera (Csillery, 1980; Daskalov and Poulos, 1994; de Vries, 1918; Ma et al., 2017; Shortess et al., 1968). Capsicum leaves do not generally contain the same dangerous alkaloids that render many solanaceous plants inedible and are often consumed as vegetables in the Philippines and other parts of Asia (Bosland, 1999). The poor vigor and high disease susceptibility of chlorophyll-related leaf mutants, such as the Capsicum lutescens, has limited their integration into commercial agriculture (Stommel and Griesbach, 2008). Mutations affecting leaf color are valuable morphological markers for breeding (e.g., male sterility) and provide novel genes for breeders as well as valuable tools in the phenotyping of Capsicum species (Ma et al., 2016; Sun et al., 2017). The distinction between lutescens mutants in Capsicum remains to be fully elucidated as a result of the current lack of study of the relationship between all lutescens accessions.

Previous research of lutescens mutants in Capsicum centered on describing their genetic characteristics and their association with male sterility, and comparatively little is known about the phytochemical constituents of the leaves of lutescent mutants (Ma et al., 2016; Rodriguez, 2017; Sun et al., 2017). A complementation study on 43 lutescens accessions conducted by Rodriguez (2017) found some hybridizations restored a dark-green leaf phenotype whereas others did not, indicating that the phenotype represented a unique genotype. Other research on a lutescent yellow-bud mutant Capsicum was also conducted recently and found that early leaves of yellow-bud mutants exhibited a very high $\mathrm{Chl} \mathrm{a} / \mathrm{b}$ ratio and elevated carotenoid concentrations, 
as well as ultrastructural signs of delayed chloroplast development (Ma et al., 2016). A similar study of the leaves of yellowgreen rice (Oryza sativa) mutants revealed the yellow-leaf mutants to have low $\mathrm{Chl} \mathrm{b}$ levels and delayed chloroplast development (Ma et al., 2017). Chang and Troughton (1972) concluded that $\mathrm{Chl} \mathrm{a} / \mathrm{b}$ ratios were dependent on environmental conditions, development stage, nutrient availability, and plant species. The correct $\mathrm{Chl} \mathrm{a} / \mathrm{b}$ ratio is vital in modulating the size of the photosynthetic antenna complex (Havaux and Tardy, 1997; Jansson, 1994; Klein et al., 1988; Oster et al., 2000).

Phytochemical phenotyping of lutescens mutants can provide the foundational evidence needed by future studies of the lutescent leaf phenotype. Phenotyping may also reveal an untapped market potential of lutescens accessions as novel photosynthetic profiles. In addition, multivariate leaf phenotyping may yield a better understanding of the regulation of chlorophyll and carotenoid biosynthesis in chloroplasts (Stommel and Griesbach, 2008). Most importantly, no model plant has been established for the study of carotenoid biosynthesis, and this group of profiled lutescent mutant accessions will be valuable to future studies of chloroplast development and carotenoid biosynthesis in vegetative tissue.

By profiling the photosynthetic pigments and obtaining International Commission on Illuminations CIEL*a*b* (CIELAB) readings for the leaves of 25 accessions of lutescens Capsicum species mutants and 'Jupiter' bell pepper (Capsicum annuum), our primary objective was to create an accurate and detailed phytochemical portrait of the lutescent phenotype. We hypothesized that all lutescent mutants would exhibit similar phenotypes and therefore would have similar pigment concentrations and CIELAB readings. In addition, we hypothesized that the lutescent mutant phenotype would be correlated with either high amounts of carotenoids or low amounts of chlorophyll.

\section{Materials and Methods}

Lutescens mutants and 'Jupiter' seed were sown on 3 May 2018. Seeds of lutescens mutants were obtained from Dr. Gabor Csillery (Budakert, Ltd., Budapest, Hungary). Three weeks later, on 24 May 2018, when the two true-leaf stage was reached, all plants were transplanted to $25.4-\mathrm{cm}$-diameter, $11.36-\mathrm{L}$ pots, each holding $\approx 11.0 \mathrm{~L}$ of soil. Three replicates per accession were transplanted together in the same $11.36-\mathrm{L}$ pot, and each plant was assigned a hyphenated numerical identifier. As a commercial benchmark cultivar that is not a lutescent mutant, 11 'Jupiter' bell pepper plants were cultivated and analyzed along with the lutescens accessions (Sieger's Seed Co., Holland, MI). 'Jupiter' bell pepper was considered the standard plant for leaf color because it produces uniform dark-green leaves, similar to 'California Wonder' (Rodriguez, 2017). Plants were germinated and grown in a temperature-controlled greenhouse using previously published propagation protocols (Coon et al., 2017). Three weeks after transplanting, on 14 June 2018, all 'Jupiter' and lutescens plants were moved to an outdoor shade house because of their poor vigor when grown in full New Mexico sunlight. All plants were then watered twice daily and fertilized with $10.0 \mathrm{~g}$ $14 \mathrm{~N}-6.1 \mathrm{P}-11.6 \mathrm{~K}$ controlled-release, general-purpose fertilizer (Osmocote; Scotts, Marysville, $\mathrm{OH}$ ) every 2 weeks until completion of the study. There was minimal insect damage, and the leaves harvested for analysis did not have symptoms.
On 3 July 2018, mature leaf samples were collected for extraction, and CIELAB readings were recorded. A colorimeter (Chroma Meter CR400; Konica Minolta Sensing America, Ramsey, NJ) obtained the CIEL*a*b* color value for each lutescens leaf sample and 'Jupiter' leaf sample. The chromameter was calibrated every 10 readings to prevent any drift in readings. The adaxial surface of mature leaves was measured. Hue angle and chroma were calculated [Hue angle $=\tan ^{-1}(\mathrm{~b} / \mathrm{a})$ and Chroma $=$ $\left(a^{2}+b^{2}\right)^{1 / 2}$ ] according to established methods (Smith, 2014).

Mature leaf tissue was collected from all replicates on 3 July 2018, when plants were mature and starting to produce fruit. Tissue was transferred from the field to the laboratory on ice at $4{ }^{\circ} \mathrm{C}$. Each leaf tissue sample was weighed immediately in the laboratory and stored at $-80^{\circ} \mathrm{C}$ until extraction. Extractions were performed under yellow lights using a previously published method with slight modifications (Guzman et al., 2012). About $1.0 \mathrm{~g}$ of fresh leaf tissue from each sample was ground in a mortar and pestle with $15 \mathrm{~mL}$ of reagent high-performance liquid chromatography (HPLC)-grade ethanol (Sigma-Aldrich, St. Louis, MO) as the extraction solvent. The ethanolic leaf extract was centrifuged at $4{ }^{\circ} \mathrm{C}$ and $1598 g_{n}$ for $10 \mathrm{~min}$, and the resulting supernatant was collected in a $40-\mathrm{mL}$ amber vial fitted with a polytetrafluoroethylene-lined cap. The remaining pellet was further extracted using an additional $15 \mathrm{~mL}$ ethanol and $20 \mathrm{~min}$ of ambient sonication using a water bath sonicator (2800 Ultrasonic Bath; Branson Ultrasonics, Danbury, CT) followed by centrifugation at $4{ }^{\circ} \mathrm{C}$ and $1598 \mathrm{~g}_{\mathrm{n}}$ for $10 \mathrm{~min}$. Last, the third round of pellet extraction with ethanol was performed and the pellet no longer contained any visible pigment. For each sample, all supernatants were pooled together in one amber glass vial. The extractions were dried down to residue under a stream of nitrogen gas. Pending analysis, dried extracts were stored in a closed amber vial at $-80^{\circ} \mathrm{C}$ blanketed in nitrogen gas and "parafilmed" to prevent oxidative degradation of photosynthetic pigments from exposure to oxygen.

The HPLC analysis on all lutescens and 'Jupiter' samples used a previously published carotenoid separation method, with adjustments to improve the separation of lutein and $\mathrm{Chl} \mathrm{b}(\mathrm{Ri}-$ chins et al., 2014). Under yellow lights, dried extracts were resuspended in $4.0 \mathrm{~mL}$ HPLC-grade isopropanol (Sigma-Aldrich) and sonicated at room temperature for $5 \mathrm{~min}$ or until all the pigment residue was dissolved. After centrifugation, the supernatant was transferred to a labeled 40-mm HPLC vial and was placed in the HPLC autosampler (717plus Autosampler; Waters Corp., Milford, MA). An HPLC system equipped with a pump (600 Controller, Waters) and a $\mathrm{C}_{30}$ carotenoid column $(4.6 \times 250$ $\mathrm{mm}, 5-\mu \mathrm{m}$ particle size, $\mathrm{C}_{30}$; YMC America, Allentown, PA) provided separation of lutein, zeaxanthin, $\beta$-carotene, Chl a, and $\mathrm{Chl} \mathrm{b.} \mathrm{The} \mathrm{solvents} \mathrm{used} \mathrm{were} \mathrm{solvent} \mathrm{A} \mathrm{[methanol:tert-butyl}$ methyl ether (MTBE):water (81:15:4)] and solvent B [methanol:MTBE:water (8:88:4)] as used previously and published by Richins et al. (2014). The solvent gradient included two linear gradients: 0 to $15 \mathrm{~min}$ from $0 \%$ to $5 \% \mathrm{~B}$ and 15 to $35 \mathrm{~min}$ from $5 \%$ to $67 \% \mathrm{~B}$. The flow rate was $3.0 \mathrm{~mL} \cdot \mathrm{min}^{-1}$, the column temperature was maintained at $25^{\circ} \mathrm{C}$, and the injection volume was $20 \mu \mathrm{L}$ for all samples. A photodiode array detector (Waters 996 Photodiode Array Detector, Waters) collected spectral absorbance data from 230 to $700 \mathrm{~nm}$. Carotenoids and chlorophylls were quantitated by extracting chromatograms at $450 \mathrm{~nm}$. Data processing software (Empower 2, Waters) was used to extract chromatograms and process peak area data. 
Serial dilutions of reference standards lutein, zeaxanthin, $\beta$-carotene, $\mathrm{Chl} \mathrm{a}$, and $\mathrm{Chl} \mathrm{b}$ (Sigma-Aldrich) were prepared using chromatography-grade solvents. Individual calibration curves were generated at $450 \mathrm{~nm}$ for each analyte. Lutein and zeaxanthin serial calibration dilutions were prepared using isopropanol whereas both $\mathrm{Chl} \mathrm{a}$ and $\mathrm{Chl} \mathrm{b}$ serial dilutions were prepared using acetone. The $\beta$-carotene serial calibration dilutions were prepared using a 50:50 hexane:isopropanol solvent. Concentrations of prepared reference standard dilutions were confirmed using a spectrophotometer (SpectraMax; M2 Molecular Devices, San Jose, CA), and all analytes were assessed spectrophotometrically at their individual maximum absorbance wavelengths.

Lutein, zeaxanthin, $\beta$-carotene, $\mathrm{Chl} \mathrm{a}$, and $\mathrm{Chl} \mathrm{b}$ in lutescens leaf tissue samples were quantitated using calibration curves prepared from analytical reference standards. To monitor the fidelity of the photodiode array detector response throughout the entire analytical data set, quality control check standards were run at regular intervals to assess the accuracy of the reference calibration curves. A lutein analytical standard was used to create quality control standards of high and low concentrations, both of which were used to assess any changes in the detector response throughout the course of analysis.

Analytical calibration curves and quantitation of photosynthetic pigments from chromatogram peak areas were prepared

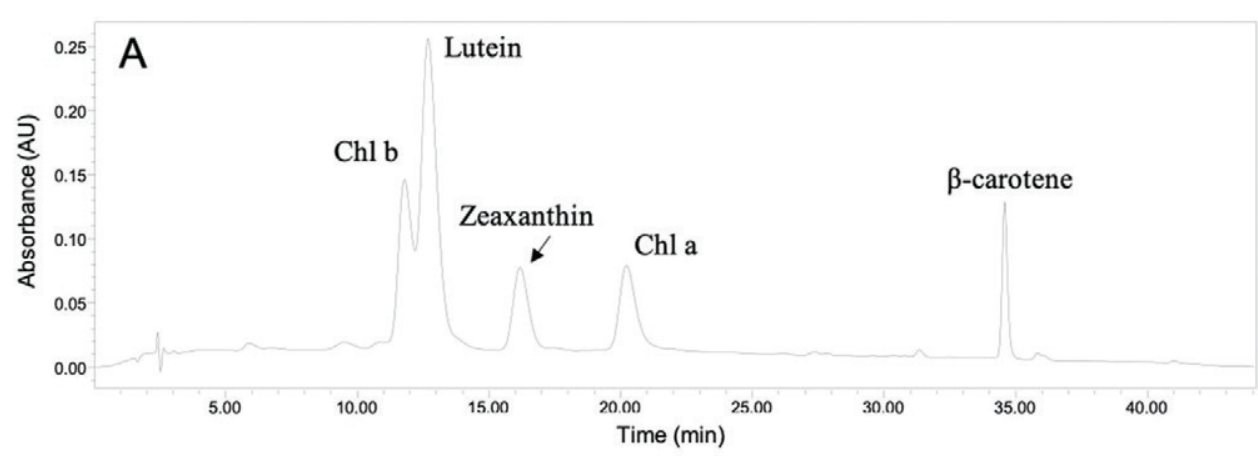

B

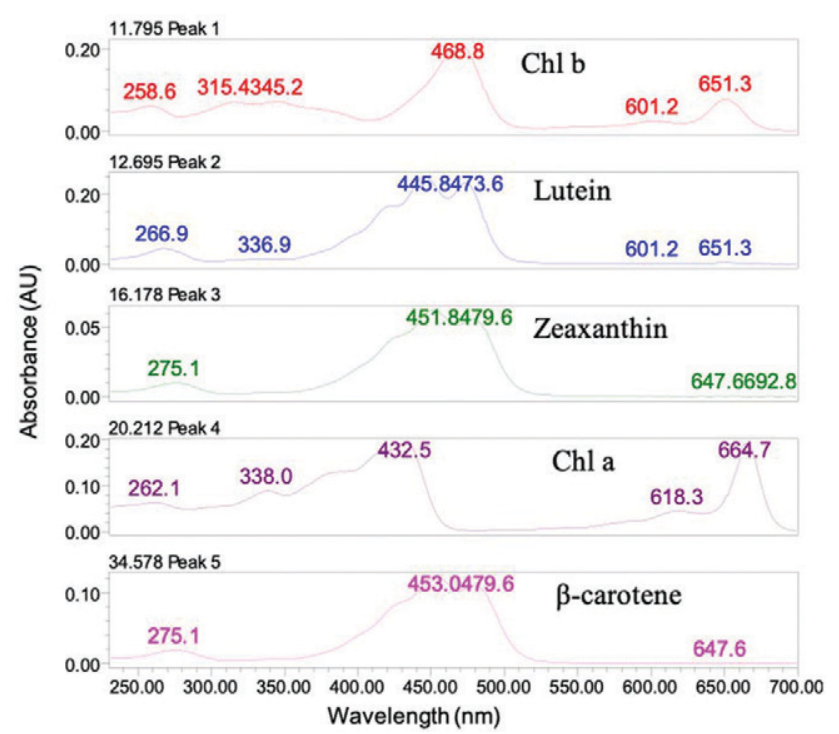

Fig. 1. (A) High-performance liquid chromatography chromatogram of lutescens Capsicum species mutant leaf pigment standards with retention times observed at $450 \mathrm{~nm}$ : chlorophyll b [(Chl b), $11.79 \mathrm{~min}]$, lutein (12.69 $\mathrm{min}$ ), zeaxanthin (16.18 $\mathrm{min})$, chlorophyll a [(Chl a), $20.21 \mathrm{~min}$ ], and $\beta$-carotene (34.58 $\mathrm{min})$. (B) Unique absorbance spectra for each standard peak, measured between the wavelengths 230 and $270 \mathrm{~nm}$. using spreadsheet software (Excel version 2008; Microsoft Corp., Redmond, WA). Data postprocessing was performed using statistical software (JMP version 14.3.0; SAS Institute, Cary, NC). Hierarchical cluster analysis (HCA) of observations was generated using JMP, as was the labeling of hierarchical clusters. After all observations were labeled with cluster markers, principal component analysis (PCA) of all variables was conducted.

\section{Results}

The HPLC methodology effectively separated $\mathrm{Chl} \mathrm{a,} \mathrm{Chl} \mathrm{b,}$ $\beta$-carotene, lutein, and zeaxanthin in a mixture of analytical standards (Fig. 1). The improved HPLC method separated Chl b successfully from lutein, with retention times of 11.8 and 12.7 min, respectively. Unique absorbance spectra of lutein, zeaxanthin, $\beta$-carotene, Chl a, and Chl b standards confirmed the identity of peaks in lutescens and 'Jupiter' extracts (Fig. 1).

All 25 lutescens accessions grown exhibited light yellowgreen mature leaves compared with 'Jupiter' (Fig. 2). Mutant lut -5 is the only lutescens accession to appear from a hybridization of $C$. frutescens $\times C$. annuum var. annuum (Csillery, 1980, 1983; Daskalov and Poulos, 1994). Lutescens accessions lut-5c and lut-5e were not included as a result of likely experimental error. In addition, several other lutescent accessions (lut-33c, lut-63, and $l u t-65)$ were also excluded from the data set as a result of a high likelihood of experimental error in sampling or analysis, bringing the final number of profiled lutescens mutant accessions to 25 .

Lutein and zeaxanthin concentrations varied in lutescens leaves (Table 1). Lutein was found consistently in greater concentrations than zeaxanthin. Interestingly, 'Jupiter' bell pepper leaves had a slightly greater average lutein concentration than the leaves of lutescent Capsicum species mutants (Table 1). Zeaxanthin was present in far lower amounts than lutein in the leaves of both 'Jupiter' and lutescens mutant accessions, and was absent entirely from lutescens accessions lut-5g, lut $-5 \mathrm{~h}$, and lut-15 (Table 1). Zeaxanthin levels in lutescens mutant accessions were less than those found in the leaves of 'Jupiter' bell pepper.

A majority of lutescens accessions, 18 out of 25 , contained $\beta$-carotene concentrations greater than the 'Jupiter' contextual benchmark average (Table 1). In fact, $\beta$-carotene was found to be the dominant leaf carotenoid in several lutescens accessions (lut-5k, lut-8, lut-11, lut-22c, and lut45fv) (Table 1).

Unidentified peaks were classified as unknown carotenoids based on their absorbance spectra. Peak areas for unknown carotenoids were summed and quantitated collectively using the 


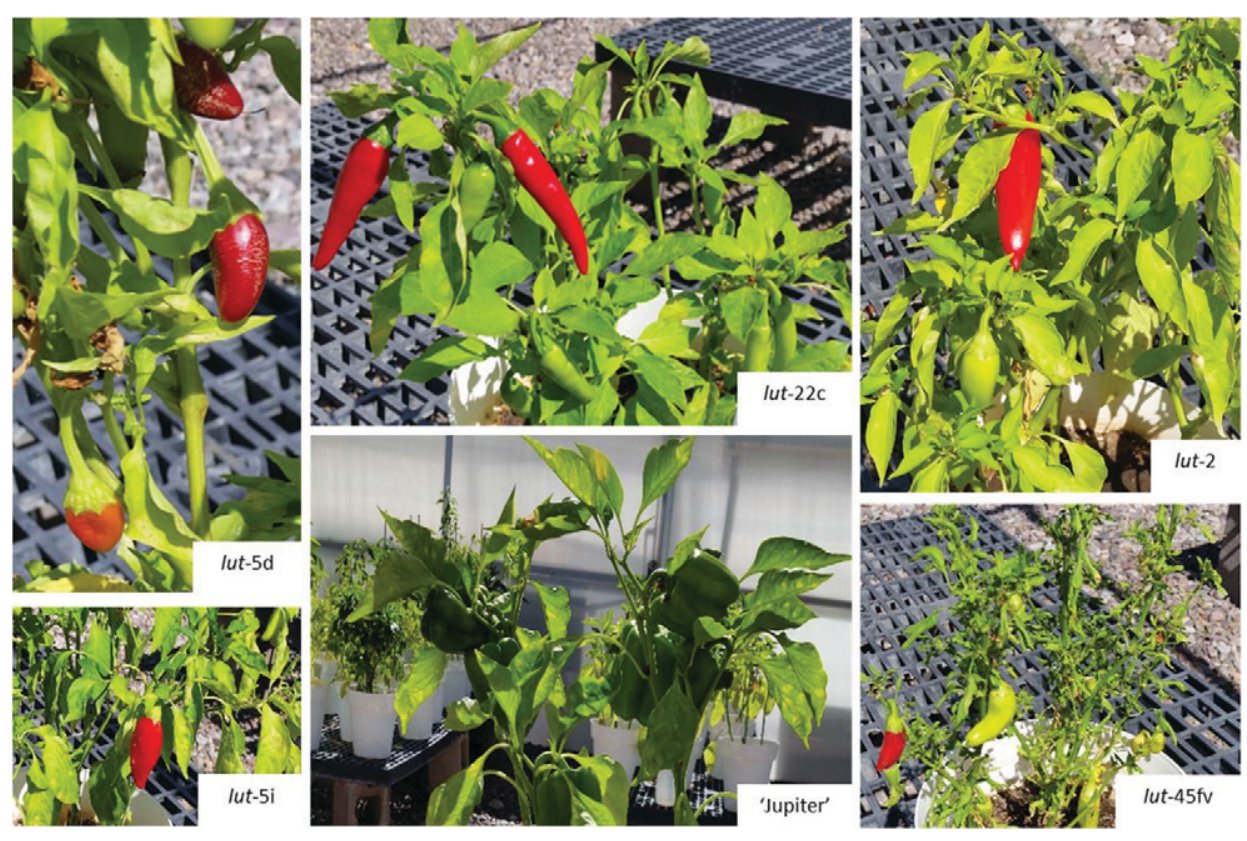

Fig. 2. Lutescens Capsicum species mutant (lut-5i, lut-5d, lut-22c, lut-2, and lut-45fv) and 'Jupiter' bell pepper plant images. Lutescens mutants exhibit light yellow-green leaves compared with the 'Jupiter' contextual benchmark. lutein calibration curve, which provided the greatest linearity in response. For this reason, concentrations of total unknown carotenoids were calculated as micrograms per gram fresh weight (FW) lutein equivalents. Overall, the concentration of total unknown carotenoids did not differ dramatically between the lutescens mutant accessions and 'Jupiter' bell pepper.

Nearly half the lutescens accessions, 12 of 25, contained concentrations of Chl a greater than $500 \mu \mathrm{g} \cdot \mathrm{g}^{-1}$ $\mathrm{FW}$, whereas nine had concentrations of Chl a greater than $600 \mu \mathrm{g} \cdot \mathrm{g}^{-1} \mathrm{FW}$ (Table 1). Concentrations of $\mathrm{Chl} \mathrm{b}$ were slightly lower in leaves of lutescens mutant accessions than in leaves of 'Jupiter' bell pepper (Table 1). The $\mathrm{Chl} \mathrm{a} / \mathrm{b}$ ratios in the leaves of lutescens mutants and 'Jupiter' bell pepper differed noticeably. The $\mathrm{Chl} a / b$ ratio was greater in leaves of lutescens mutants than in 'Jupiter' (Fig. 3). All 25

Table 1. Mean concentrations of leaf pigments (chlorophyll a, chlorophyll b, lutein, zeaxanthin, and other unidentified carotenoids) and chlorophyll a/b ratios measured in lutescens Capsicum species mutants and 'Jupiter' bell pepper $(\mathrm{n}=3)$.

\begin{tabular}{|c|c|c|c|c|c|c|c|}
\hline \multirow{2}{*}{$\begin{array}{l}\text { Lutescens } \\
\text { accession }\end{array}$} & \multicolumn{6}{|c|}{ Concn, mean $\pm \mathrm{SE}\left(\mu \mathrm{g} \cdot \mathrm{g}^{-1} \mathrm{FW}\right)$} & \multirow[b]{2}{*}{ Chlorophyll a/b ratio $\pm \mathrm{SE}$} \\
\hline & Chlorophyll a & Chlorophyll b & Lutein & Zeaxanthin & $\beta$-Carotene & Unidentified carotenoids $^{\mathrm{z}}$ & \\
\hline lut-2 & $377.6 \pm 50.5$ & $142.3 \pm 16.6$ & $47.3 \pm 4.2$ & $1.2 \pm 0.6$ & $36.3 \pm 3.2$ & $35.3 \pm 4.5$ & $2.7 \pm 0.1$ \\
\hline lut-3 & $707.6 \pm 43.2$ & $299.8 \pm 7.6$ & $104.0 \pm 4.8$ & $2.7 \pm 0.1$ & $70.4 \pm 3.3$ & $79.5 \pm 4.2$ & $2.4 \pm 0.2$ \\
\hline$l u t-4^{\mathrm{y}}$ & $367.5 \pm 0$ & $95.0 \pm 0$ & $44.2 \pm 0$ & $1.5 \pm 0$ & $36.8 \pm 0$ & $36.7 \pm 0$ & $3.9 \pm 0$ \\
\hline lut $-5 \mathrm{a}$ & $435.3 \pm 11.7$ & $175.9 \pm 11.3$ & $62.2 \pm 4$ & $2.3 \pm 0.4$ & $42.4 \pm 1.9$ & $56.0 \pm 4.8$ & $2.5 \pm 0.2$ \\
\hline lut-5d & $294.1 \pm 96.6$ & $111.6 \pm 5$ & $48.7 \pm 3.7$ & $2.1 \pm 1.2$ & $39.6 \pm 4.7$ & $37.9 \pm 7.1$ & $2.6 \pm 0.8$ \\
\hline lut $-5 \mathrm{~g}$ & $523.5 \pm 44.2$ & $175.5 \pm 11.7$ & $63.9 \pm 4.1$ & n.d. & $50.8 \pm 5.5$ & $64.0 \pm 4.8$ & $3.0 \pm 0.1$ \\
\hline lut $-5 \mathrm{~h}$ & $668.8 \pm 83$ & $275.1 \pm 5.7$ & $102.6 \pm 3$ & n.d. & $59.2 \pm 7.8$ & $75.4 \pm 6.9$ & $2.4 \pm 0.3$ \\
\hline lut-5i & $687.0 \pm 25.2$ & $244.9 \pm 5.5$ & $84.1 \pm 2.3$ & $1.8 \pm 0$ & $57.1 \pm 1.3$ & $77.8 \pm 6.3$ & $2.8 \pm 0.1$ \\
\hline lut $-5 \mathrm{k}$ & $353.1 \pm 122.3$ & $134.9 \pm 19.5$ & $47.2 \pm 5.2$ & $0.8 \pm 0.8$ & $54.1 \pm 10.5$ & $40.0 \pm 11.6$ & $2.6 \pm 1.0$ \\
\hline lut-8 & $652.4 \pm 50.8$ & $193.4 \pm 15.4$ & $60.6 \pm 3.6$ & $2.2 \pm 0.1$ & $71.1 \pm 5$ & $64.7 \pm 6.9$ & $3.4 \pm 0.2$ \\
\hline lut-11 & $587.2 \pm 43.4$ & $180.4 \pm 6.4$ & $59.8 \pm 3.8$ & $1.6 \pm 0.8$ & $64.1 \pm 2.7$ & $67.0 \pm 2.8$ & $3.3 \pm 0.2$ \\
\hline lut -15 & $185.0 \pm 67.5$ & $77.9 \pm 3.6$ & $28.8 \pm 4.2$ & n.d. & $25.9 \pm 2.9$ & $24.5 \pm 4.4$ & $2.4 \pm 0.5$ \\
\hline lut-16 & $592.9 \pm 117.4$ & $228 \pm 45.7$ & $76.8 \pm 16.8$ & $0.8 \pm 0.8$ & $57.9 \pm 2.6$ & $77.1 \pm 24.4$ & $2.6 \pm 0.1$ \\
\hline lut $-22 \mathrm{c}$ & $378.3 \pm 40.8$ & $88.1 \pm 5.4$ & $36.1 \pm 1.8$ & $2.0 \pm 0.1$ & $45.8 \pm 2.5$ & $41.6 \pm 4.4$ & $4.3 \pm 0.2$ \\
\hline lut $-22 \mathrm{a}$ & $342.2 \pm 16.4$ & $90.2 \pm 3.3$ & $47.6 \pm 3.5$ & $0.8 \pm 0.8$ & $40.0 \pm 1.4$ & $47.0 \pm 1.8$ & $3.8 \pm 0.2$ \\
\hline lut-24a & $359.3 \pm 108.7$ & $131.5 \pm 19.1$ & $52.4 \pm 7$ & $1.4 \pm 0.7$ & $39.1 \pm 5.9$ & $45.2 \pm 7.8$ & $2.7 \pm 0.7$ \\
\hline lut-27 & $681.2 \pm 95.7$ & $313.5 \pm 10.4$ & $113.5 \pm 7.7$ & $0.9 \pm 0.9$ & $46.1 \pm 7.1$ & $89.2 \pm 8.4$ & $2.2 \pm 0.2$ \\
\hline lut-31 & $349.7 \pm 87.1$ & $121.1 \pm 25.1$ & $45.9 \pm 8.1$ & $1.4 \pm 0.8$ & $36.4 \pm 7.1$ & $43.1 \pm 12.9$ & $2.9 \pm 0.2$ \\
\hline lut-39 & $711.4 \pm 141.3$ & $306.2 \pm 12.6$ & $105.1 \pm 9.6$ & $2.2 \pm 0.1$ & $64.0 \pm 4.1$ & $87.3 \pm 11.8$ & $2.3 \pm 0.4$ \\
\hline lut-40 & $287.1 \pm 48.4$ & $108.9 \pm 6.9$ & $42.4 \pm 4.8$ & $0.7 \pm 0.7$ & $38.6 \pm 4.4$ & $32.0 \pm 3.7$ & $2.6 \pm 0.3$ \\
\hline lut $-45 \mathrm{fv}$ & $371.8 \pm 70$ & $121.4 \pm 9$ & $44.7 \pm 1.9$ & $0.6 \pm 0.6$ & $50.1 \pm 3.1$ & $45.0 \pm 3.2$ & $3.1 \pm 0.4$ \\
\hline lut-47 & $338.1 \pm 24$ & $65.3 \pm 5.5$ & $61.5 \pm 5$ & $2.7 \pm 0.3$ & $41.7 \pm 3.7$ & $60.3 \pm 5.1$ & $5.2 \pm 0.3$ \\
\hline lut-51 & $763.2 \pm 112.2$ & $315.4 \pm 13.8$ & $101.4 \pm 4.9$ & $1.3 \pm 0.7$ & $61.8 \pm 3.4$ & $95.3 \pm 12.4$ & $2.4 \pm 0.3$ \\
\hline lut -57 & $635.1 \pm 40.1$ & $216.5 \pm 1.8$ & $78.5 \pm 2.7$ & $0.9 \pm 0.5$ & $60.6 \pm 1.9$ & $85.3 \pm 7.3$ & $2.9 \pm 0.2$ \\
\hline lut-59 & $749.8 \pm 21.2$ & $278.7 \pm 2.9$ & $95.7 \pm 1.8$ & $2.1 \pm 0.2$ & $52.6 \pm 0.9$ & $101.9 \pm 5.6$ & $2.7 \pm 0.1$ \\
\hline 'Jupiter' & $434.4 \pm 41.7$ & $238.5 \pm 13.1$ & $84.7 \pm 4.9$ & $2.9 \pm 0.1$ & $31.8 \pm 2.0$ & $57.2 \pm 5.4$ & $1.8 \pm 0.1$ \\
\hline
\end{tabular}

${ }^{\mathrm{z}}$ Unidentified carotenoids calculated in lutein equivalents.

${ }^{\mathrm{y}}$ Accession lut-4 $(\mathrm{n}=1)$.

$\mathrm{FW}=$ fresh weight; n.d. $=$ not detected. 
accessions of lutescens mutants exhibited $\mathrm{Chl} \mathrm{a} / \mathrm{b}$ ratios $\geq 2.0$, eight of which exhibited ratios $\geq 3.0$ (Fig. 3). Accessions lut-22c and lut-47 exhibited very high mean $\mathrm{Chl}$ a/b ratios (Fig. 3). Overall, the leaves of lutescens mutants were found to have greater amounts of $\mathrm{Chl} \mathrm{a}$ and lower amounts of $\mathrm{Chl} \mathrm{b}$ than 'Jupiter' bell pepper leaves, causing the $\mathrm{Chl} \mathrm{a} / \mathrm{b}$ ratios of lutescens mutant accessions to be greater than those of 'Jupiter'.

CIELAB $\left(\mathrm{L}^{*} \mathrm{a}^{*} \mathrm{~b}\right)$ color values were obtained from the leaves of 25 lutescens mutant accessions as well as from the 'Jupiter' contextual benchmark (Table 2). Lutescens mutant accessions had a wide array of values in CIELAB color space (Table 2). The entire range of lutescens leaf colors plotted in the CIELAB color space encompassed that of 'Jupiter' bell pepper (Table 2). As expected, CIELAB readings could not predict the concentration of any individual pigment because leaf tissue contains multiple pigments with reflective regions that overlap (Fig. 4). Although differences in hue angle and chroma values between 'Jupiter' and lutescent leaf phenotypes were not readily apparent from CIELAB values alone, both hue and chroma were important variables for PCA.

An HCA of observations was created using the common Ward method in JMP (Fig. 5). Observations from 'Jupiter' leaves are clustered together, as can be expected. Interestingly, observations from lutescent mutants cluster into several distinct, major groups (Fig. 5). For example, accessions lut-3, lut-5h, lut-16, lut27, lut-39, lut-51, and lut-59 are clustered together tightly but are set apart from all other lutescens clusters (Fig. 5).

PCA provided another vital tool to visualize and interpret relationships stemming from the large number of pigment variables (Rencher, 2002). Seven clusters provided the greatest number of cluster groups that still maintained all 'Jupiter' observations as one group, which was established as the baseline estimate. The use of 'Jupiter' leaves as a contextual benchmark was vital for this reason. The seven clusters selected from the hierarchical cluster of observations (Fig. 5) were marked with different symbols before being plotted in the PCA (Fig. 6).

The clusters of observations marked in the HCA were grouped tightly in the PCA plot and were separated primarily along principal component 1 (PC 1) (Fig. 6A). 'Jupiter' observations also grouped; but, in contrast, were separated from all lutescens accessions mostly along principal component 2 (PC 2) (Fig. 6A). The sum of PC 1 and PC 2 in the PCA loading plot was $80.2 \%$, meaning a two-dimensional representation of the data accounted for $80.2 \%$ of the observation variability (Fig. $6 \mathrm{~B})$.

PC 1 is composed of a high positive correlation with concen-

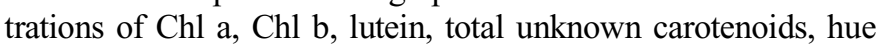
angle, and $\beta$-carotene; and high negative correlations with saturation, chroma, and, last, $\mathrm{Chl}$ a/b ratio (Fig. 6B). PC 2 was composed primarily of a positive correlation with zeaxanthin content, and a negative correlation with $\beta$-carotene content and the $\mathrm{Chl} \mathrm{a} / \mathrm{b}$ ratio (Fig. 6B).

'Jupiter' leaves were distinguished from all lutescens leaves by increased levels of zeaxanthin, decreased concentrations of $\beta$-carotene, and a lower $\mathrm{Chl} \mathrm{a/b}$ ratio, as expected in normal leaves. It is also clear that lutescens accessions, separated by PC 1 , have a significant range of phenotypic profiles, and correlate both positively and negatively with PC 1 . In fact, PC 1 distinguished six observational groups of lutescent phenotype profiles. Two of the major groups correlate negatively with PC 1 and two correlate positively with PC 1 .
The PCA indicates that leaves of lutescent mutants, typically with greater $\mathrm{Chl} \mathrm{a} / \mathrm{b}$ ratios and greater $\beta$-carotene concentrations than 'Jupiter' leaves, are distinguished from commercial 'Jupiter' leaves by variables known to be related to light stress

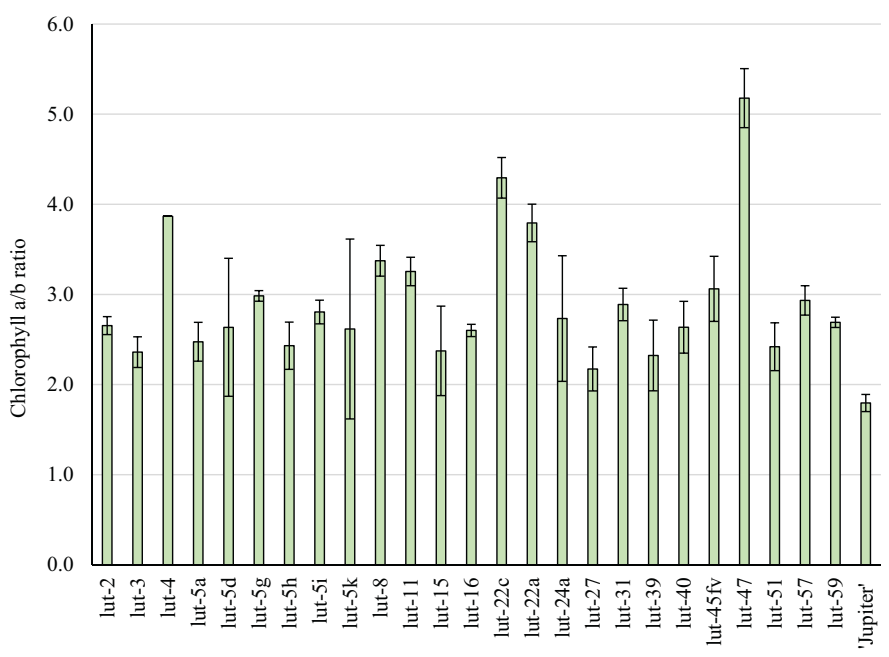

Fig. 3. Chlorophyll a/b mean ratios $\pm \mathrm{SE}$ in leaves of lutescens Capsicum species mutants and 'Jupiter' bell pepper. Leaves of lutescens mutants exhibit consistently greater chlorophyll a/b ratios than 'Jupiter' leaves. For all accessions, $\mathrm{n}=3$; for accession lut $-4, \mathrm{n}=1$.

Table 2. Mean International Commission on Illuminations CIEL*a*b* $\left(\mathrm{L}^{*}, \mathrm{a}^{*}\right.$, and $\left.\mathrm{b}^{*}\right)$ measurements, hue angle, and chroma for lutescens Capsicum species mutants and 'Jupiter' bell pepper leaves $(\mathrm{n}=3)$. Accession lut-4 $(\mathrm{n}=1)$.

\begin{tabular}{|c|c|c|c|c|c|}
\hline Lutescens accession & $\mathrm{L}^{*}$ & $a^{*}$ & $\mathrm{~b}^{*}$ & Hue angle $\left({ }^{\circ}\right)$ & Chroma \\
\hline lut -2 & 45.18 & -20.05 & 29.70 & -55.98 & 35.84 \\
\hline lut-3 & 41.33 & -16.09 & 22.83 & -54.80 & 27.93 \\
\hline lut-4 & 51.78 & -20.30 & 35.94 & -60.52 & 41.29 \\
\hline lut-5a & 46.26 & -19.22 & 30.28 & -57.36 & 35.88 \\
\hline lut-5d & 53.10 & -21.65 & 38.33 & -60.54 & 44.02 \\
\hline lut $-5 \mathrm{~g}$ & 48.04 & -18.39 & 29.63 & -58.05 & 34.89 \\
\hline lut $-5 \mathrm{~h}$ & 41.19 & -15.87 & 23.31 & -55.67 & 28.20 \\
\hline lut-5i & 46.14 & -17.55 & 28.68 & -58.34 & 33.65 \\
\hline lut-5k & 45.39 & -18.57 & 28.17 & -56.60 & 33.74 \\
\hline lut-8 & 43.42 & -19.24 & 29.07 & -56.48 & 34.86 \\
\hline lut-11 & 45.96 & -19.79 & 31.73 & -57.99 & 37.40 \\
\hline lut -15 & 57.18 & -22.28 & 43.00 & -62.59 & 48.44 \\
\hline lut-16 & 38.88 & -12.83 & 17.23 & -53.22 & 21.49 \\
\hline lut-22c & 50.70 & -20.22 & 35.59 & -60.39 & 40.94 \\
\hline lut $-22 \mathrm{a}$ & 51.90 & -19.96 & 37.27 & -61.80 & 42.28 \\
\hline lut-24 & 49.13 & -17.11 & 31.36 & -60.93 & 35.79 \\
\hline lut-27 & 42.21 & -14.77 & 21.94 & -56.04 & 26.45 \\
\hline lut-31 & 50.94 & -20.21 & 35.89 & -60.60 & 41.19 \\
\hline lut-39 & 42.55 & -15.19 & 22.22 & -55.60 & 26.92 \\
\hline lut-40 & 52.21 & -21.18 & 39.00 & -61.39 & 44.39 \\
\hline lut $-45 \mathrm{fv}$ & 48.94 & -17.63 & 29.74 & -59.33 & 34.57 \\
\hline lut-47 & 58.55 & -19.32 & 45.29 & -66.86 & 49.26 \\
\hline lut -51 & 42.81 & -16.13 & 23.34 & -55.24 & 28.39 \\
\hline lut-57 & 46.28 & -17.41 & 28.09 & -58.18 & 33.05 \\
\hline lut-59 & 38.86 & -14.80 & 19.96 & -53.47 & 24.86 \\
\hline 'Jupiter' 18c 1172 & 48.66 & -18.73 & 32.62 & -60.05 & 37.62 \\
\hline 'Jupiter' 18c 1173 & 48.52 & -18.20 & 32.58 & -60.73 & 37.33 \\
\hline 'Jupiter' $18 \mathrm{c} 1175$ & 51.68 & -19.14 & 36.52 & -62.33 & 41.24 \\
\hline
\end{tabular}




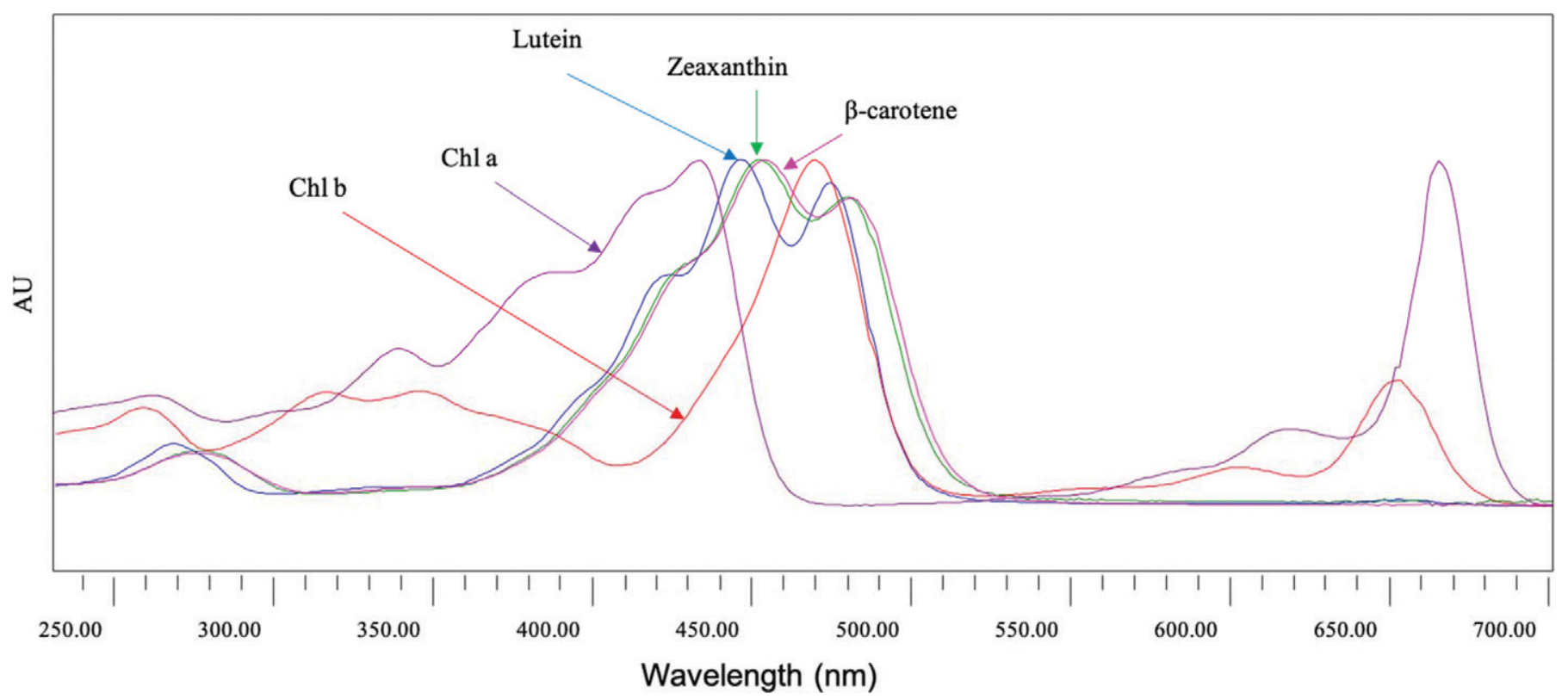

Fig. 4. High-performance liquid chromatography photodiode array detector absorbance spectra of pigment standards [lutein, zeaxanthin, $\beta$-carotene, and chlorophyll (Chl) a and b] measured from 230 to $700 \mathrm{~nm}$.

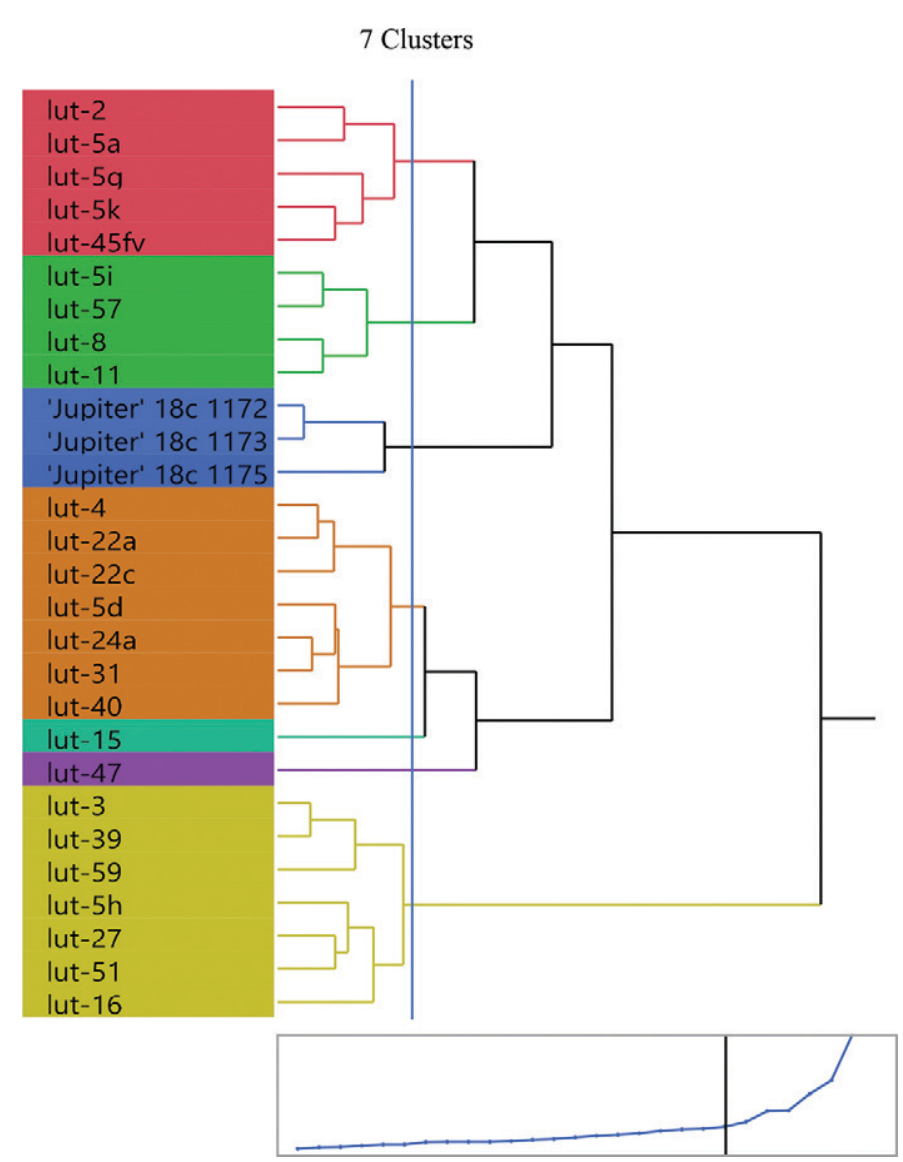

Fig. 5. Hierarchical clustering dendrogram of leaf pigments from lutescens Capsicum species mutants and 'Jupiter' bell pepper indicating several clusters of lutescens accessions. and delayed development (Keyhaninejad et al., 2012; Ma et al., 2016). The PCA also provides evidence that lutescens mutants have a wide array of phenotypes.

\section{Discussion}

Lutescens mutants of Capsicum species did not have greater levels of lutein or zeaxanthin than chlorophyll, rejecting the hypothesis that the lutescens phenotype is a result of elevated xanthophyll concentrations alone. Based on the results of the PCA, lutescens accessions were distinguished the most from one another by a combination of color variables by the CIELAB color measurements and individual pigment concentrations. All lutescens accessions were distinguished from 'Jupiter' benchmark leaves by variables known to be related to light stress and delayed development - namely, a negative correlation with $\mathrm{Chl} a / b$ ratio and $\beta$-carotene content (Chang and Troughton, 1972; Keyhaninejad et al., 2012; Ma et al., 2016). It remains to be determined whether the clustering of observations in our observational study of lutescens leaves represents unique individual phenotypes.

An explanation for these findings is that lutescens genetic mutations cause delays in chloroplast development and decrease photosynthetic activity, which is supported by the high $\mathrm{Chl} a / b$ ratios and high $\beta$-carotene presence observed in lutescens mutants. Stunted chloroplast development in lutescens mutants may result in photoinhibition at a lower light intensity than 'Jupiter' leaves, as developmental delays limit the number of photocenters in lutescens mutants (Ma et al., 2016). This effect likely stimulates lutescent mutant leaves to increase $\beta$-carotene production in response to high light stress, even at low light intensities, protecting their limited photocenters from photooxidative damage. 

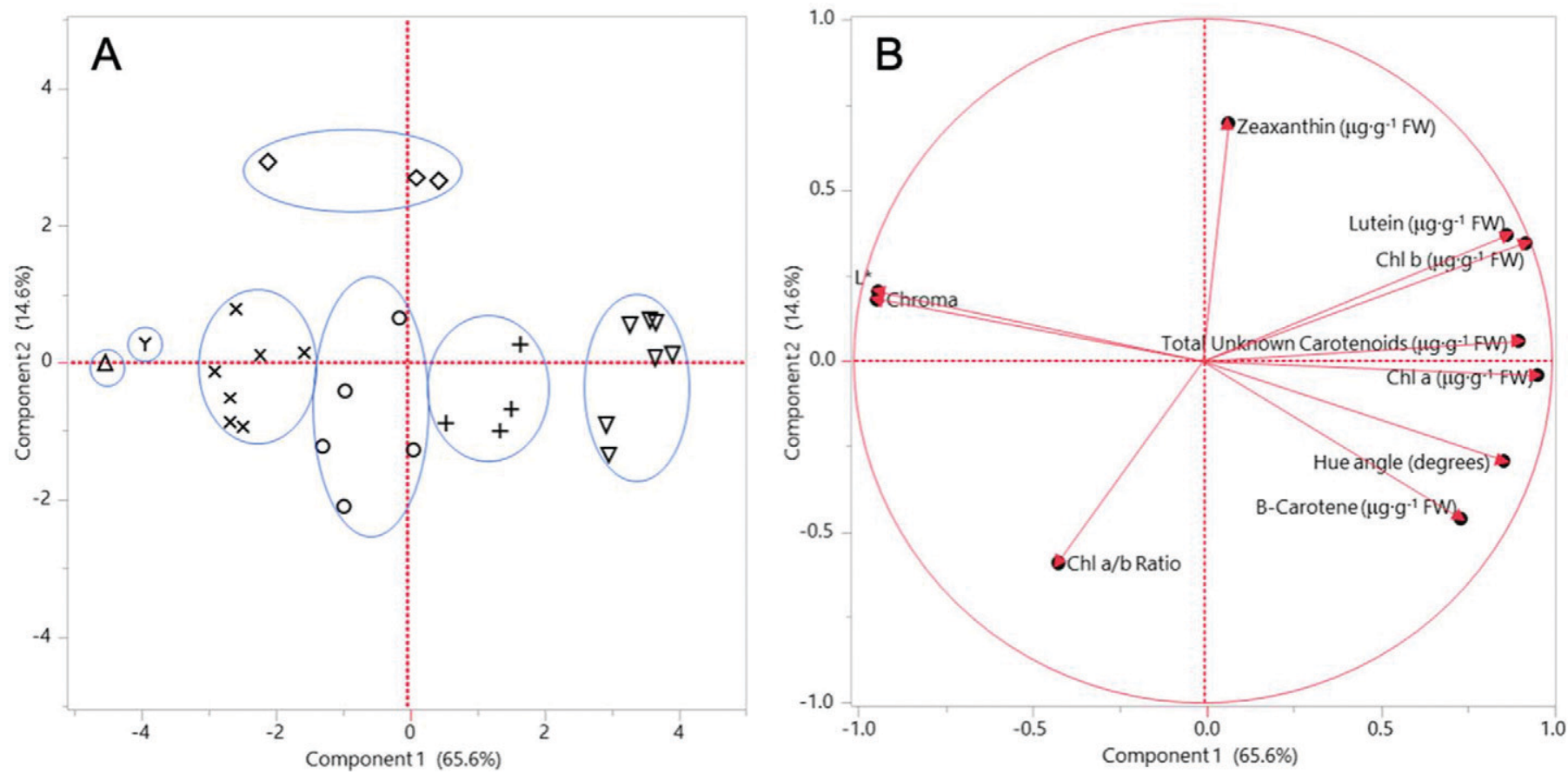

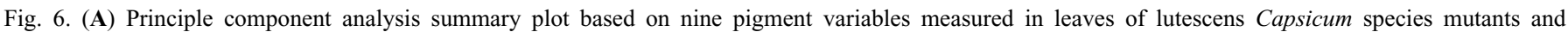
'Jupiter' bell pepper indicated seven groups of lutescens Capsicum species mutant accessions. Four major groups, one 'Jupiter' bell pepper group, and two minor lutescens groups were separated primarily along principle component 1. Principle component 2 separated 'Jupiter' from all lutescens accessions. (B) Principal component 1 and 2 explained $65.6 \%$ and $14.6 \%$ of the observed variation, respectively. Chl, chlorophyll; FW, fresh weight.

Delayed vegetative development also offers an explanation for the multiple phenotypic groups observed in the PCA. It is possible that delayed development causes variable maturation rates among mutant accessions - meaning, different accessions sampled at the same age after germination may be, in fact, at different developmental growth stages. Previous research has consistently failed to determine a standard leaf color for all lutescens mutants, supporting the speculation that different mutant accessions express different phenotypes (Csillery, 1980, 1983, 1985; Rodriguez, 2017). Alternatively, some mutant accessions have also been found to be caused by the same allelic mutation (Rodriguez, 2017). Chloroplast ultrastructural analysis is needed to determine whether each lutescent accession truly has a unique individual phenotype or whether multiple accessions in our study exhibited the same several phenotypes at varying developmental stages. This study is the first to phenotype Capsicum lutescens both phytochemically and colorimetrically, as well as the first to use a multivariate approach to lutescens mutant phenotyping, ultimately providing valuable insights into the study of the lutescens mutant phenotype in Capsicum and its possible connection to chloroplast development.

\section{Literature Cited}

Bosland, P.W. 1999. Chiles: A gift from a fiery god. HortScience 34: 809-881, doi: 10.21273/hortsci.34.5.809.

Buchanan, B.B. 2015. Biochemistry and molecular biology of plants. Wiley, Hoboken, NJ.

Chang, F.H. and J.H. Troughton. 1972. Chlorophyll a/b ratios in C3and C4-plants. Photosynthetica 6:57-65.

Coon, D., D.W. Barchenger, and P.W. Bosland. 2017. Evaluation of dwarf ornamental chile pepper cultivars for commercial greenhouse production. HortTechnology 27:128-131, doi: 10.21273/HORTTECH03452-16.
Csillery, G. 1980. Gene mapping of the pepper needs more initiatives (contributions to the gene list). Proc. 4th Eucarpia Meeting of Capsicum Working Group, 17-19 May 1980, Wageningen, The Netherlands. p. 5-9.

Csillery, G. 1983. New Capsicum mutants found on seedling, growth type, leaf, flower and fruit. Proc. 5th Eucarpia Meeting of Capsicum and Eggplant Working Group, 4-7 July 1983, Plovdiv, Bulgaria. p. $127-130$

Csillery, G. 1985. Abnormal segregation ratio in a 'lutescens' hybrid in Capsicum baccatum. Capsicum Eggplant Nwsl. 4:43.

Daskalov, S. and J.M. Poulos. 1994. Updated Capsicum gene list. Capsicum Eggplant Nwsl. 13:15-26.

de Vries, H. 1918. Mutations of Oenothera suaveolens DESF. Genetics $3: 1-26$.

Gomez-Garcia, M. del R. and N. Ochoa-Alejo. 2013. Biochemistry and molecular biology of carotenoid biosynthesis in chili peppers (Capsicum spp.). Intl. J. Mol. Sci. 14:19025-19053, doi: 10.3390/ ijms 140919025.

Guzman, I., G.G. Yousef, and A.F. Brown. 2012. Simultaneous extraction and quantitation of carotenoids, chlorophylls, and tocopherols in Brassica vegetables. J. Agr. Food Chem. 60:7238-7244, doi: $10.1021 / \mathrm{jf302475d}$.

Havaux, M. and F. Tardy. 1997. Thermostability and photostability of photosystem II in leaves of the chlorina-f2 barley mutant deficient in light-harvesting chlorophyll $\mathrm{a} / \mathrm{b}$ protein complexes. Plant Physiol. 113:913-923, doi: 10.1104/pp.113.3.913.

Jansson, S. 1994. The light-harvesting chlorophyll a b-binding proteins. BBA Bioenergetics 1184:1-19, doi: 10.1016/0005-2728(94) 90148-1.

Keyhaninejad, N., R.D. Richins, and M.A. O'Connell. 2012. Carotenoid content in field-grown versus greenhouse-grown peppers: Different responses in leaf and fruit. HortScience 47:852-855, doi: 10.21273/HORTSCI.47.7.852.

Klein, R.R., P.E. Gamble, and J.E. Mullet. 1988. Light-dependent accumulation of radiolabeled plastid-encoded chlorophyll a-apoproteins 
requires chlorophyll a. Plant Physiol. 88:1246-1256, doi: 10.1104/ pp.88.4.1246.

Ma, Z.H., G.S. Sun, C.W. Zhang, Q. Wang, Z.L. Dai, C.Q. Sun, and Y.P. Pan. 2016. Chlorophyll content, chloroplast ultrastructure and transcriptome analysis in wild-type and yellow-bud-mutant hot peppers. J. Agr. Sci. Technol. 18:1065-1078.

Ma, X., S. Xiaoqiu, C. Li, R. Huan, C. Sun, Y. Wang, F. Xiao, Q. Wang, P. Chen, F. Ma, K. Zhang, P. Wang, and X. Deng. 2017. Map-based cloning and characterization of the novel yellow-green leaf gene ys83 in rice (Oryza sativa). Plant Physiol. Biochem. 111:19, doi: 10.1016/j.plaphy.2016.11.007.

Oster, U., R. Tanaka, A. Tanaka, and W. Rudiger. 2000. Cloning and functional expression of the gene encoding the key enzyme for chlorophyll b biosynthesis (CAO) from Arabidopsis thaliana. Plant J. 21: 305-310, doi: 10.1046/j.1365-313X.2000.00672.x.

Rencher, A.C. 2002. Methods of multivariate analysis. Wiley, Hoboken, NJ, doi: 10.2307/1268907.

Richins, R.D., J. Kilcrease, L. Rodriguez-Uribe, and M.A. O'Connell. 2014. Carotenoid extraction and quantification from
Capsicum annuum. Bio Protoc. 4:e1256, doi: 10.21769/ BioProtoc. 1256.

Rodriguez, K.K. 2017. Characterization of lutescens mutants in chile pepper (Capsicum spp.). New Mexico State University, Las Cruces, NM, MS thesis.

Shortess, D.K., J.E. Wright, and W.D. Bell. 1968. The lutescent mutant in maize: I. Inheritance patterns and environmental effects. Genetics 58:227-235.

Smith, S.D. 2014. Quantifying color variation: Improved formulas for calculating hue with segment classification. Appl. Plant Sci. 2: 1300088, doi: 10.3732/apps. 1300088 .

Stommel, J.R. and R.J. Griesbach. 2008. Inheritance of fruit, foliar, and plant habit attributes in Capsicum. J. Amer. Soc. Hort. Sci. 133: 396-407, doi: 10.1017/S0022109000004178.

Sun, G.S., Z.L. Dai, P.W. Bosland, Q. Wang, C.Q. Sun, Z.C. Zhang, and Z.H. Ma. 2017. Characterizing and marker-assisting a novel chili pepper (Capsicum annuum L.) yellow bud mutant with cytoplasmic male sterility. Genet. Mol. Res. 16(1), doi: $10.4238 /$ gmr16019459. 